\title{
Does Optic Nerve Sheath Diameter Ultrasonography Permit Accurate Detection of Real-Time Changes in ICP?
}

Daniel N. Lesky; Christopher K. Schott, MD, MS, RDMS

\section{One-time optic nerve sheath diameter ultrasonography permits risk-stratifying patients with suspected elevated intracranial pressure (ICP). Some data suggest that the technique also may be useful for detecting real-time changes in ICP.}

\section{Case Scenarios}

\section{Case 1}

While working abroad in a resource-limited environment, a patient was brought in after falling and hitting his head. Initially, the patient was awake and alert, but he gradually became minimally responsive, with a Glasgow Coma Scale score of 9. Your facility did not have computed tomography (CT) or magnetic resonance imaging (MRI), but did have a point-of-care ultrasound (US) machine. You measured the patient's optic nerve sheath diameter (ONSD) with the US and found a diameter of $4.5 \mathrm{~mm}$ in each eye. With this clinical change, you wondered if repeat US scans to detect increasing intracranial pressure (ICP) would represent changes in the patient's condition.

\section{Case 2}

A patient who presented with an intracranial hemorrhage was treated with hypertonic saline and was awaiting neurosurgical placement of an extraventicular drain. During this time, a resident who was on a US rotation asked you if she would be able to detect changes in the patient's ICP using US rather than placing an invasive device. How do you respond?

In adults, ICP is normally 10 to $15 \mathrm{~mm} \mathrm{Hg}$. It may be pathologically increased in several life-threatening conditions, including traumatic brain injury (TBI), subarachnoid hemorrhage, central venous thrombosis, brain tumor, and abscess. It is also increased by nonacute pathology, such as idiopathic intracranial hypertension (IIH), which also is known as pseudotumor cerebri. In patients with acute pathology, ICP above $20 \mathrm{~mm} \mathrm{Hg}$ is generally considered an indication for treatment. ${ }^{1}$ Indications for ICP monitoring in TBI include positive CT findings, patient age greater than 40 years, systemic hypotension, or abnormal flexion/extension in response to pain. ${ }^{2}$ Other reasons to monitor ICP include the management of pseudotumor cerebri or after ventriculoperitoneal shunt surgery. ${ }^{3}$

Unfortunately, current methods of ICP monitoring have significant drawbacks and limitations. The gold standard of ICP monitoring-measurement using an in-

Mr Lesky was a medical student, University of Pittsburgh School of Medicine, Pennsylvania, at the time this article was written. Dr Schott is an assistant professor, department of critical care medicine and emergency medicine, University of Pittsburgh and University of Pittsburgh Medical Center, VA Pittsburgh Health Care Systems, Pennsylvania. The opinions expressed in this paper do not reflect those of the Veterans Health Administration. 
traventricular catheter-increases the risks of infection and hemorrhage, requires the skill of a neurosurgeon, and may be contraindicated due to coagulopathy or thrombocytopenia. It also cannot be done in a prehospital setting and only to a limited extent in the ED. ${ }^{4}$

Computed tomography scans and MRI can assess elevated ICP, but these tests are expensive, may increase patient radiation exposure, require patient transport, and may not always detect raised ICP. In the appropriate clinical context, signs present on physical examination, such as decorticate/decerebrate posturing, papilledema, or fixed/dilated pupils, may be highly suggestive of an increased ICP, but sensitivity and specificity are inadequate. Delay in diagnosis is also a drawback of imaging and physical examination, as findings may not present until ICP has been persistently elevated.

Given the disadvantages of current means of assessing elevated ICP, several noninvasive methods of measuring ICP are being investigated. These include such techniques as transcranial Doppler, electroencephalogram, pupillometry, and ONSD measurements. ${ }^{5}$ This article reviews current applications of ultrasonography measurements of the ONSD in assessing elevations in ICP.

\section{ONSD US}

Assessment of ICP via measurement of the ONSD has attracted increasing attention, particularly in emergency medicine. Measurements of the ONSD are possible with

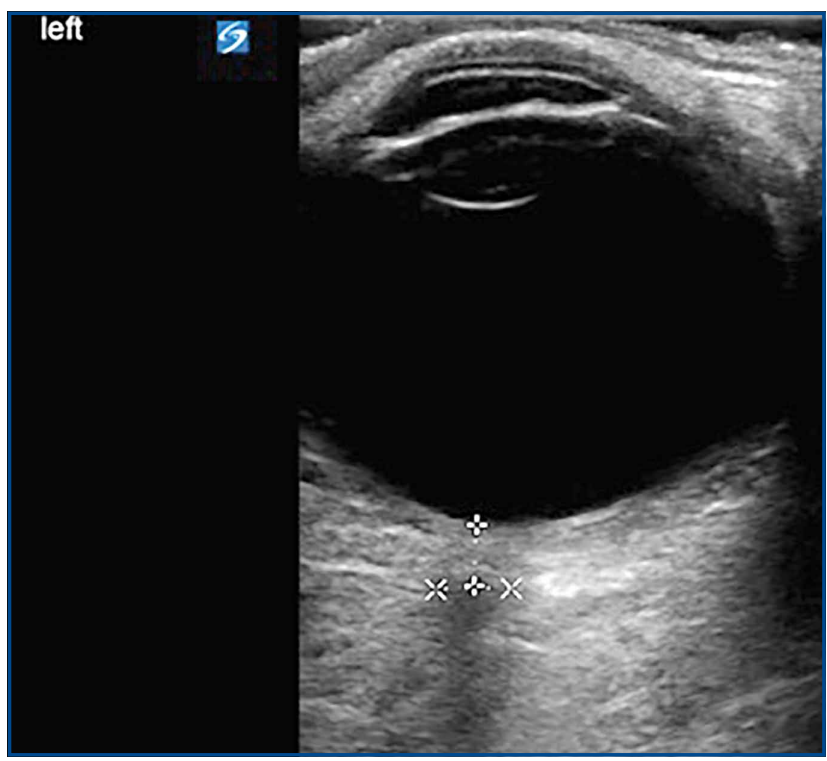

Figure 1. The appearance of a normal eye and optic nerve sheath seen in a transverse view with bedside ultrasound.
CT, MRI, and US. Of these modalities, ONSD US has attracted the most interest, due to its low cost, wide availability, and rapidity. It does not require patient transport, and does not expose a patient to additional radiation. In addition, ONSD US has been utilized in low-resource settings, and may be particularly useful in prehospital and mass-casualty situations. ${ }^{6}$

The underlying relationship between ONSD and ICP is a result of the enclosure of the subarachnoid space by the ONS. Increased ICP leads to expansion of the ONS, particularly at $3 \mathrm{~mm}$ behind the globe, in the retrobulbar compartment (Figures 1 and 2). ${ }^{7}$

Unfortunately, it is not possible to precisely determine ICP from an ONSD measurement, because baseline ONSD values and elasticity vary significantly within the population..$^{4,8}$ As a result, ONSD US has been investigated mostly for its ability to detect qualitative changes-particularly as a screen for elevated ICP. Optic nerve sheath diameter has high discriminative value in its ability to distinguish normal from elevated ICP. In a meta-analysis, Dubourg et $\mathrm{al}^{9}$ showed that the technique had an area under the summary receiver-operating curve of 0.94 , signifying excellent test accuracy to diagnose elevated ICPs.

Researchers have attempted to determine a threshold value of ONSD that would serve as a clinically useful predictor of elevated ICP. Currently, this value ranges from 4.8 to $5.9 \mathrm{~mm}$, depending on the study $; 5 \mathrm{~mm}$ is commonly used clinically as a threshold. ${ }^{10}$

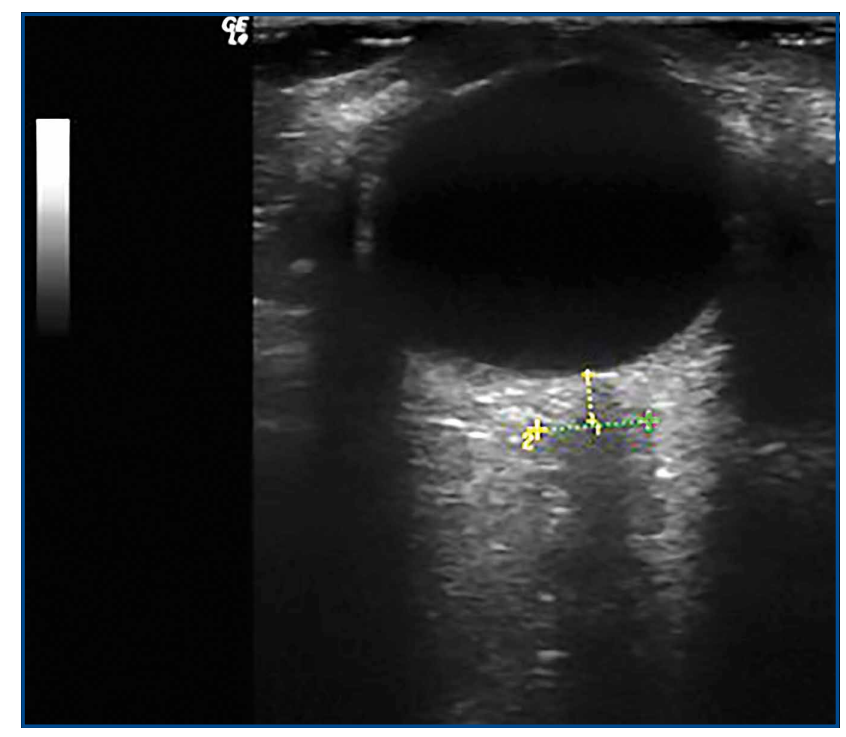

Figure 2. The appearance of an abnormal optic nerve sheath diameter at the proper point $3 \mathrm{~mm}$ posterior to the retina, seen in a transverse view with bedside ultrasound. 


\section{Using ONSD US to Monitor Rapid Changes in ICP}

While the use of the ONSD technique to screen for elevated ICP is relatively well established, the use of ONSD US to track acute changes in ICP is not as well studied. Serial tracking of acute changes could be useful in a patient at risk for intracranial hypertension secondary to trauma, to monitor the results of treating a patient with $\mathrm{IIH}$, or after ventriculoperitoneal shunt placement. ${ }^{3}$

\section{In Vivo Data}

In 1993, Tamburrelli et al $^{11}$ performed the first ONSD intrathecal infusion study, using A-scan sonography, and concluded that there was a "direct, biphasic, positive relation between diastolic intracranial pressure and optic nerve diameters" and that the data showed "rapid changes of optic nerve diameters in response to variation of intracranial pressure."

In 1997, Hansen and Helmke ${ }^{12}$ recorded ONSD versus ICP data in the first intrathecal infusion test to use Bscan mode sonography. Ultrasonography was performed at 2- to 4-minute intervals. Their data demonstrated a linear relationship between ICP and ONSD over a particular cerebrospinal fluid pressure interval. They noted that "this interval differed between patients: ONS dilation commenced at pressure thresholds between $15 \mathrm{~mm} \mathrm{Hg}$ and $30 \mathrm{~mm} \mathrm{Hg}$ and in some patients saturation of the response (constant ONSD) occurred between $30 \mathrm{~mm} \mathrm{Hg}$ and $40 \mathrm{~mm} \mathrm{Hg."}$

The slope of ONSD versus ICP curve varied considerably by patient, making it impossible to infer an absolute ICP value from an ONSD without prior knowledge of the patient's ratio. Similar to the data from Tamburrelli et al, ${ }^{11}$ Hansen and Helmke ${ }^{12}$ also found that there was no lag in ONSD response to ICP: "Within this interval, no temporal delay of the ONS response was noted."

The only study comparing real-time ONSD data to gold-standard measurements of rapidly changing ICP in humans was performed by Maissan et $\mathrm{al}^{13}$ in 2015 . This study involved a cohort of 18 patients who had suffered TBI and had intraparenchymal probes inserted. Because ICP rises transiently during endotracheal tube suctioning due to irritation of the trachea, the increase and subsequent decrease after suctioning was an ideal time to perform ONSD measurements and compare them to simultaneous gold-standard ICP measurements. The ONSD US measurements were performed 30 to 60 seconds prior to suctioning, during suctioning, and 30 to 60 seconds after suctioning.
Even during this very rapid time course, a strong correlation between ICP and ONSD measurements was demonstrated. The R2 value was 0.80 . There was no perceptible "lag" in ONSD change; changes in ICP were immediately reflected in ONSD. Notably, an absolute change of less than 8 to $10 \mathrm{~mm} \mathrm{Hg}$ in ICP did not affect ONSD, which is consistent with data collected by Hansen and Helmke. ${ }^{12}$

\section{Therapeutic Lumbar Puncture for IIH}

There are two case reports of ONSD US measurements being taken pre- and postlumbar puncture (LP) in patients with IIH. In the first, in 1989 Galetta et al ${ }^{14}$ used A-scan US to measure pre- and post-LP ONSD in a woman with papilledema secondary to IIH. They found a significant reduction in ONSD bilaterally "within minutes" of performing the LP. ${ }^{14}$

The second case report was published in 2015 by Singleton et al. ${ }^{15}$ They recorded ONSD measurements 30 minutes pre- and post-LP in a woman who presented to the ED with symptoms from elevated ICP. After reduction of pressure via LP, they recorded a significant reduction in ONSD bilaterally. ${ }^{15}$

\section{Cadaver Data}

Hansen et $\mathrm{al}^{16}$ evaluated the distensibility and elasticity of the ONS using postmortem optic nerve preparations. The ONSD was recorded 200 seconds after each pressure increase, which was long enough to achieve stable diameters. They found a linear correlation between pressure increases of 5 to $45 \mathrm{~mm} \mathrm{Hg}$ and ONSD. This would suggest a potential positively correlated change in ONSD with in vivo changes in ICP. However, this still needs further clinical study to better assess measurable changes in living patients.

\section{Conclusion}

Published data have consistently demonstrated that changes in ICP are rapidly transmitted to the optic nerve sheath and that there does not appear to be a temporal lag in the ONSD. Based on in vivo data, the relationship between ICP and ONSD appears to be linear only over a range of moderately elevated ICP. According to Hansen and Helmke, ${ }^{12}$ this range starts at approximately 18 to $30 \mathrm{~mm} \mathrm{Hg}$, and ends at approximately 40 to $45 \mathrm{~mm} \mathrm{Hg}$. Maissan et $\mathrm{al}^{13}$ observed similar findings: "At low levels, ICP changes (8-10 $\mathrm{mm} \mathrm{Hg}$ ) do not affect the ONSD."

There is still need for additional research to validate 
and refine these findings. Only one study has compared gold-standard ICP measurements with ONSD US measurements in real time, ${ }^{13}$ and the literature on ONSD US in tracking ICP after therapeutic LP in IIH consists of only two case reports.

Thus, with some caveats, ONSD US appears to permit qualitative tracking of ICP in real time. This supports its use in situations where a patient may have rapidly changing ICP, such as close monitoring of patients at risk for elevated ICP in a critical care setting, and response to treatment in patients with IIH.

\section{References}

1. Stocchetti N, Maas AI. Traumatic intracranial hypertension. N Engl J Med. 2014;370(22):2121-2130.

2. Brain Trauma Foundation; American Association of Neurological Surgeons; Congress of Neurological Surgeons; et al. Guidelines for the management of severe traumatic brain injury. VI. Indications for intracranial pressure monitoring. J Neurotrauma. 2007;24(Suppl 1):S37-S44

3. Choi SH, Min KT, Park EK, Kim MS, Jung JH, Kim H. Ultrasonography of the optic nerve sheath to assess intracranial pressure changes after ventriculo-peritoneal shunt surgery in children with hydrocephalus: a prospective observational study. Anaesthesia. 2015;70(11):1268-1273.

4. Kristiansson $\mathrm{H}$, Nissborg E, Bartek J Jr, Andresen M, Reinstrup P, Romner B. Measuring elevated intracranial pressure through noninvasive methods: a review of the literature. J Neurosurg Anesthesiol. 2013;25(4):372-385.

5. Rajajee V, Thyagarajan P, Rajagopalan RE. Optic nerve ultrasonography for detection of raised intracranial pressure when invasive monitoring is unavailable. Neurol India. 2010;58(5):812-813.

6. Robba C, Baciqaluppi S, Cardim D, Donnelly J, Bertuccio A, Czosnyka
M. Non-invasive assessment of intracranial pressure. Acta Neurol Scand. 2016;134(1):4-21.

7. Hansen HC, Helmke K. The subarachnoid space surrounding the optic nerves. An ultrasound study of the optic nerve sheath. Surg Radiol Anat. 1996;18(4):323-328.

8. Hansen HC, Lagrèze W, Krueger O, Helmke K. Dependence of the optic nerve sheath diameter on acutely applied subarachnoidal pressure - an experimental ultrasound study. Acta Ophthalmol. 2011;89(6):e528-e532.

9. Dubourg J, Javouhey E, Geeraerts T, Messerer M, Kassai B. Ultrasonography of optic nerve sheath diameter for detection of raised intracranial pressure: a systematic review and meta-analysis. Intensive Care Med. 2011;37(7):1059-1068.

10. Kimberly HH, Shah S, Marill K, Noble V. Correlation of optic nerve sheath diameter with direct measurement of intracranial pressure. Acad Emerg Med. 2008;15(2):201-204.

11. Tamburrelli C, Anile C, Mangiola A, Falsini B, Palma P. CSF dynamic parameters and changes of optic nerve diameters measured by standardized echography. In: Till P, ed. Ophthalmic Echography 13: Proceedings of the 13th SIDUO Congress, Vienna, Austria, 1990; vol 55. Dordrecht, Netherlands: Springer Netherlands; 1993:101-109.

12. Hansen HC, Helmke K. Validation of the optic nerve sheath response to changing cerebrospinal fluid pressure: ultrasound findings during intrathecal infusion tests. J Neurosurg. 1997;87(1):34-40.

13. Maissan IM, Dirven PJ, Haitsma IK, Hoeks SE, Gommers D, Stolker RJ. Ultrasonographic measured optic nerve sheath diameter as an accurate and quick monitor for changes in intracranial pressure. $J$ Neurosurg. 2015;123(3)743-747.

14. Galetta S, Byrne SF, Smith JL. Echographic correlation of optic nerve sheath size and cerebrospinal fluid pressure. J Clin Neuroophthalmol. 1989;9(2):79-82.

15. Singleton J, Dagan A, Edlow JA, Hoffmann B. Real-time optic nerve sheath diameter reduction measured with bedside ultrasound after therapeutic lumbar puncture in a patient with idiopathic intracranial hypertension. Am J Emerg Med. 2015;33(6):860.e5-e7.

16. Hansen HC, Lagrèze W, Krueger O, Helmke K. Dependence of the optic nerve sheath diameter on acutely applied subarachnoidal pressure-an experimental ultrasound study. Acta Ophthalmol. 2011;89(6):e528-e532. 